\title{
Challenges of managing Enterobacter endocarditis; a review of two cases
}

\author{
Piyasiri DLB, Priyarangani WKAP, Kariyawasam I, Wijeweera D, Bodinayake CK, \\ Kodikaraarachchi RWK, Madurawe RD, Sathananthan PP, Jayasekara JVGM \\ Teaching Hospital Karapitiya, Galle, Sri Lanka.
}

\author{
Correspondence: Dr. D L Bhagya Piyasiri \\ e-mail: bhagyapiyasiri@yahoo.co.uk
}

\begin{abstract}
Introduction
Infective endocarditis (IE) carries significant mortality and morbidity and Streptococci and Staphylococci are the common causative organisms Apart from the HACEK group (Haemophilus, Actinobacillus, Cardiobacterium, Eikenella and Kingella), IE due to other gram-negatives are rarely reported. Risk factors of IE by non-HACEK group include intravenous drug abuse, healthcare contact and the presence of prosthetic cardiac devices (1). Here we report a review of two cases of complicated Enterobacter endocarditis.
\end{abstract}

\section{Case Report 1}

A 46-year old male drug addict from Elpitiya, Galle with the history of rheumatic carditis in childhood presented in February 2016 with sudden onset slurred speech and left upper limb numbness for one day and intermittent fever with loss of appetite for 2 weeks.

He was febrile $\left(103^{\circ} \mathrm{F}\right)$ and had a systolic murmur in the mitral area. Total white cell count (WCC) was $16.3 \times 10^{9} / \mathrm{L}$ with $76 \%$ neutrophils. C-Reactive Protein (CRP) was $202 \mathrm{mg} / \mathrm{L}$ and ESR was $40 \mathrm{~mm} / 1^{\text {st }} \mathrm{hr}$. IV penicillin and gentamicin were started empirically after 3 blood cultures. 2D Echocardiogram revealed $10 \times 10 \mathrm{~mm}$ size mitral valve vegetation. Within 24 hours incubation, all blood cultures became positive for Gram-negative bacilli and IV penicillin was replaced with IV ceftriaxone. The isolate was a lactose fermenter on MacConkey agar and gave acid/acid reaction with gas production in the Kligler iron agar test. All had the same colony morphology and the same antibiotic sensitivity pattern showing sensitivity to amikacin, meropenem and ceftriaxone and resistance to ampicillin, co-amoxiclav and cefuroxime. The isolate was identified as Enterobacter cloacae by the typical colony morphology, Kligler iron agar reaction and the RapID commercial kit of biochemicals.

On the $4^{\text {th }}$ day, duplex scan was performed due to pain in the left calf and it revealed left femoral artery embolus and embolectomy was done. Contrast enhanced CT scan showed right basal ganglia infraction. By this time, fever had not responded to ceftriaxone and patient was clinically deteriorating with rising total white cell count $(23.9 \mathrm{x}$ $\left.10^{9} / \mathrm{L}\right)$.

After identification of the organism as Enterobacter cloacae by the $5^{\text {th }}$ day, IV meropenem was started in place of ceftriaxone and gentamicin was continued. Following the change, there was a dramatic response of fever and the clinical condition improved with reduction of vegetation size $(6 \times 8 \mathrm{~mm})$ and inflammatory markers. After 4 weeks of treatment, total white cell count was $8.34 \times 10^{9} / \mathrm{L}$ with CRP of $8 \mathrm{mg} / \mathrm{L}$, along with the vegetation size of $2 \times 3 \mathrm{~mm}$ and the clinical response. Antibiotics were continued for 8 weeks in total.

\section{Case Report 2}

A 73-year old previously healthy, male admitted with intermittent low grade fever, loss of appetite, loss of weight and fatiguability for 3 weeks. During his 10 days of hospital stay, he was investigated extensively for anaemia with haemoglobin $9 \mathrm{~g} / \mathrm{dL}$ and the bone marrow biopsy was done to exclude any myelodysplastic syndrome. 2D echocardiogram was normal. He had culture positive urinary tract infection with Enterococcus faecalis and fever subsided with co-amoxiclav. 
After 2 months he presented with the same symptoms and this time there was a grade IV pansystolic murmur at the cardiac apex. On the day of admission he developed gross painless haematuria. 2D echocardiogram revealed multiple vegetations attached to posterior leaflet of the mitral valve with the largest measuring $13 \times 8 \mathrm{~mm}$. Two of the three blood samples became positive within 24 hours of collection for Enterobacter aerogenes. The organism was sensitive to co-amoxiclav, cefuroxime, ceftazidime, ceftriaxone, cotrimoxazole, ciprofloxacin, amikacin, gentamicin and meropenem while resistant to ampicillin.

His haemoglobin was $4.8 \mathrm{~g} / \mathrm{dL}$ with platelet count of $96 \times 10^{9} / \mathrm{L}$ and white cell count was within normal limits with lymphocytic predominance. ESR was $161 \mathrm{~mm} / 1^{\text {st }}$ hour and CRP was $96 \mathrm{mg} / \mathrm{L}$. Blood picture showed bicytopenia with normochromic normocytic anaemia and thrombocytopenia. His bone marrow biopsy showed hypoplastic marrow. Immunohistochemistry studies excluded the possibility of a lymphoma. Meanwhile his AntiNuclear Antibody (ANA), Double Stranded DNA (DsDNA), Serology for hepatitis and HIV were negative and liver and renal functions remained within normal limits. His chest radiograph, CT brain and ultrasound scan of the abdomen showed no abnormality.

The patient was initially treated with IV ceftriaxone $2 \mathrm{~g}$ daily and IV gentamicin for 16 days. During the first 12 days his fever and other symptoms settled with reduction of CRP from 96 to 50 and then 33). However, fever recurred on $13^{\text {th }}$ day and CRP increased from 33 to 56). Septic screening were negative while repeat echocardiogram showed only a slight reduction of the size of the largest vegetation (From $13 \times 8 \mathrm{~mm}$ to $7 \times 5 \mathrm{~mm}$ ). Suspecting probable cephalosporin resistance, ceftriaxone was replaced with IV meropenem $1 \mathrm{~g} 8$ hourly the patient showed a marked improvement. He developed an episode of neutropenia on $9^{\text {th }}$ day of IV meropenem which completely recovered with GM-CSF $5 \mu \mathrm{g} / \mathrm{kg}$ for three days.

After completing 8 weeks of meropenem, his CRP and white cell count became normal. However, the echo findings suggested persistent vegetations without further regression. He refused to have IV antibiotics further and was counseled to undergo vegetectomy and valvular repair in one month.

\section{Discussion}

Enterobacter spp. are a member of the family Enterobacteriaceae and are mucoid lactose fermenters mimicking Klebsiella morphology. They are motile and have the acid slant with acid butt and gas reaction in the Kligler iron agar test. It is known to cause pyelonephritis, intra-abdominal infections, chest infections, bone, joint and soft tissue infections and sepsis including various types of nosocomial infections but only very few cases of endocarditis have been described in the medical literature.

According to an Enterobacter endocarditis case report published by Tunkel et al (1992), about 10 out of 17 cases had mitral valve involvement while four others had aortic valve disease (2). Among the published cases the mortality rate was $44.4 \%$ (2). Enterobacter are capable of forming biofilms and of secreting some cytotoxins such as enterotoxins, hemolysins, and pore-forming toxins which can be important for its pathogenicity (4).

It is a great challenge to treat IE caused by Enterobacter with the various mechanisms of antibiotic resistance of the organism and with the toxic effects of the antibiotics in the long term therapy. Combination therapy with a beta lactam (penicillins, cephalosporins or carbapenems) and an aminoglycoside is effective for non-HACEK group endocarditis (2). Despite the in-vitro sensitivity to cefotaxime or ceftriaxone, some bacteria such as Enterobacter species can develop inducible resistance to $3^{\text {rd }}$ generation cephalosporins due to their inherent AmpC beta-lactam gene resulting in therapeutic failures $(2,3)$.

In these patients we initially continued IV ceftriaxone according to our primary ABST. However, after we confirmed the identity of the organism and with the poor clinical response we replaced ceftriaxone with IV meropenem and IV gentamicin for 8 weeks which led to a remarkably good outcome. Most of the patients with Gram negative infective endocarditis need surgical intervention as in our second case, though a few might recover with antibiotic therapy alone.

Therapeutic success in infective endocarditis often shows how vigilant and knowledgeable the clinician is. Patient's understanding and corporation is required as it needs a longer duration of antibiotics which can result in toxicity, side effects, risk of 
secondary infections, increased cost and poor compliance. The clinician needs to anticipate such problems and detect them early with strict monitoring of haematological and biochemical parameters. Regular echocardiograms, communications with other members of the team and patient also required.

\section{References}

1. Moon J, Smith T, Sahud AG, Bhanot N, An unusual etiology of infective endocarditis: Enterobacter cloacae. Jinfect Chemother 2012; 18(6): 925-30.

2. Tunkel AR, Fisch MJ, Schlein A, Scheld WM, Enterobacter endocarditis, Scand J Infect Dis, 1992; 24(2): 233-40.
3. Yoshino Y, Okugawa S, Kimura S, Makita E, Seo K, Koga I, Matsunaga N, Kitazawa T, Ota Y, Infective endocarditis due to Enterobacter cloacae resistant to third and fourth-generation cephalosporins, Journal of Microbiology, Immunology and Infection, 2015; 48(2): 226-8.

4. Anne Davin-Regli, Jean-Marie Pagès. Enterobacter aerogenes and Enterobacter cloacae; versatile bacterial pathogens confronting antibiotic treatment. Front Microbiol 2015; 6: 392. 